\title{
A new mathematical model for Zika virus transmission
}

\author{
Shahram Rezapour ${ }^{1,2,3}$, Hakimeh Mohammadi $4^{*}$ and Amin Jajarmi ${ }^{5,6}$
}

"Correspondence:

hakimeh.mohammadi.02@gmail.com

${ }^{4}$ Department of Mathematics,

Miandoab Branch, Islamic Azad

University, Miandoab, Iran

Full list of author information is

available at the end of the article

\section{Springer}

\begin{abstract}
We present a new mathematical model for the transmission of Zika virus between humans as well as between humans and mosquitoes. In this way, we use the fractional-order Caputo derivative. The region of the feasibility of system and equilibrium points are calculated, and the stability of equilibrium point is investigated. We prove the existence of a unique solution for the model by using the fixed point theory. By using the fractional Euler method, we get an approximate solution to the model. Numerical results are presented to investigate the effect of fractional derivative on the behavior of functions and also to compare the integer-order derivative and fractional-order derivative results.
\end{abstract}

MSC: Primary 34A08; secondary 65P99; 49J15

Keywords: Equilibrium point; Fractional derivative; Euler method; Numerical simulation; Zika virus

\section{Introduction}

Zika virus was first detected in monkeys in 1947, and the first cases of Zika virus infection were reported in 1952 in Uganda and the Republic of Tanzania. Between 1960 and 1980, there were few human infections with Zika virus in Asia and Africa, until in 2007 in the Yap Islands, then in 2013 in the France Polynesia and the Pacific Ocean, and in 2015 in Brazil the widespread outbreak of the virus was reported. Subsequently, it has spread to other countries around the world, so far Zika virus infection has been recorded in 86 countries.

Fever, rash, conjunctivitis, muscle and joint pain, malaise, or headache are symptoms of this disease. According to the report of World Health Organization (WHO), Zika virus infection during pregnancy can cause infants to be born with microcephaly and other congenital malformations, known as congenital Zika syndrome. Also, at the result of this disease, other complications of pregnancy including preterm birth and miscarriage may happen. In adults and children, Zika virus infection is associated with neurologic complications including Guillain-Barre syndrome, neuropathy, and myelitis.

Zika is a virus that is spread mostly by Aedes mosquitoes. Besides that, this virus can be transmitted from mother to baby during pregnancy or around the time of birth. Also, it can spread through blood transfusions and sexual contact.

(c) The Author(s) 2020. This article is licensed under a Creative Commons Attribution 4.0 International License, which permits use sharing, adaptation, distribution and reproduction in any medium or format, as long as you give appropriate credit to the original author(s) and the source, provide a link to the Creative Commons licence, and indicate if changes were made. The images or other third party material in this article are included in the article's Creative Commons licence, unless indicated otherwise in a credit line to the material. If material is not included in the article's Creative Commons licence and your intended use is not permitted by statutory regulation or exceeds the permitted use, you will need to obtain permission directly from the copyright holder. To view a copy of this licence, visit http://creativecommons.org/licenses/by/4.0/. 
The study of diseases dynamics is a dominating theme for many biologists and mathematicians (see, for example, [1-3]). It has been studied by many researchers that fractional extensions of mathematical models of integer order represent the natural fact in a very systematic way such as in the approach of Baleanu et al. [4-17]. Studies on the methods of solving fractional differential equations and the application of fractional systems have also been conducted [18-24]. In recent years, fractional-order derivatives have expanded and have been widely used in modeling real-world phenomena and investigating the process of disease transmission and control (see, for example, [25-35]). Also, some studies in the biological models with fractional-order derivative have been conducted in recent years [36-39]. During last years researchers have been using some mathematical models to simulate the transmission of Zika virus [40-43].

In mathematical models of Zika virus transmission it is assumed that the virus is usually transmitted from mosquitoes to humans, while according to WHO, in addition to the transmission through mosquitoes, Zika virus is transmitted through infected blood as well as through sexual contact with an infected person. In this article, we consider a mathematical model based on both ways of transmitting the virus. Also, according to the good results of fractional-order derivative in the modeling of real-world phenomena in recent years, we use Caputo fractional-order derivative instead of the integer-order derivative in this model.

The structure of the paper is as follows. In Sect. 2 some basic definitions and concepts of fractional calculus are recalled. The transmission model of Zika virus with fractionalorder derivative is presented in Sect. 3, and the equilibrium points and the reproduction number are calculated. The existence and uniqueness of solution for the system are proved in Sect. 4. Numerical method and numerical results are presented in Sect. 5.

\section{Preliminaries}

In this section, we recall some basic concepts of fractional differential calculus.

Definition 2.1 ([44]) For an integrable function $g$, the Caputo derivative of fractional order $v \in(0,1)$ is given by

$$
{ }^{C} D^{v} g(t)=\frac{1}{\Gamma(m-v)} \int_{0}^{t} \frac{g^{(m)}(v)}{(t-v)^{v-m+1}} d v, \quad m=[v]+1 .
$$

Also, the corresponding fractional integral of order $v$ with $\operatorname{Re}(v)>0$ is given by

$$
{ }^{C} I^{v} g(t)=\frac{1}{\Gamma(v)} \int_{0}^{t}(t-v)^{v-1} g(v) d v
$$

Definition $2.2([45,46])$ For $g \in H^{1}(c, d)$ and $d>c$, the Caputo-Fabrizio derivative of fractional order $v \in(0,1)$ for $g$ is given by

$$
{ }^{C F} D^{v} g(t)=\frac{M(v)}{(1-v)} \int_{c}^{t} \exp \left(\frac{-v}{1-v}(t-v)\right) g^{\prime}(v) d v \text {, }
$$

where $t \geq 0, M(v)$ is a normalization function that depends on $v$ and $M(0)=M(1)=1$. If $g \notin H^{1}(c, d)$ and $0<v<1$, this derivative for $g \in L^{1}(-\infty, d)$ is given by

$$
{ }^{C F} D^{v} g(t)=\frac{v M(v)}{(1-v)} \int_{-\infty}^{d}(g(t)-g(v)) \exp \left(\frac{-v}{1-v}(t-v)\right) d v
$$


Also, the corresponding $C F$ fractional integral is presented by

$$
{ }^{C F} I^{v} g(t)=\frac{2(1-v)}{(2-v) M(v)} g(t)+\frac{2 v}{(2-v) M(v)} \int_{0}^{t} g(v) d v
$$

The Laplace transform is one of the important tools in solving differential equations that are defined below for two kinds of fractional derivative.

Definition 2.3 ([44]) The Laplace transform of Caputo fractional differential operator of order $v$ is given by

$$
L\left[{ }^{C} D^{v} g(t)\right](s)=s^{\nu} L g(t)-\sum_{i=0}^{m-1} s^{\nu-i-1} g^{(i)}(0), \quad m-1<v \leq m \in N,
$$

which can also be obtained in the form

$$
L\left[{ }^{C} D^{v} g(t)\right]=\frac{s^{m} L[g(t)]-s^{m-1} g(0)-s^{m-1} g^{\prime}(0)-\cdots-g^{(m-1)}}{s^{m-v}} .
$$

\section{Model formulation}

In this section, we provide a mathematical model for the transmission of Zika virus using the Caputo derivative of fractional order. We divide the human population $N_{h}$ into two groups: susceptible people $S_{h}$ and infected people $I_{h}$ so that $N_{h}=S_{h}+I_{h}$. Similarly, we divide the total number of mosquitoes $N_{m}$ into two groups: susceptible mosquitoes $S_{m}$ and infected mosquitoes $I_{m}$ so that $N_{m}=S_{m}+I_{m}$. To describe the mechanism of the spread of Zika virus, we consider the compartmental mathematical model as follows:

$$
\left\{\begin{array}{l}
\frac{d S_{h}}{d t}=\Lambda_{h}-\beta_{1} S_{h} I_{h}-\beta_{2} S_{h} I_{m}-k_{1} S_{h} \\
\frac{d t_{h}}{d t}=\beta_{1} S_{h} I_{h}+\beta_{2} S_{h} I_{m}-k_{1} I_{h} \\
\frac{d S_{m}}{d t}=\Lambda_{m}-\mu S_{m} I_{h}-k_{2} S_{m} \\
\frac{d I_{m}}{d t}=\mu S_{m} I_{h}-k_{2} I_{m}
\end{array}\right.
$$

with the initial conditions $S_{h}(0)=S_{0 h}, I_{h}(0)=I_{0 h}, S_{m}(0)=S_{0 m}, I_{m}(0)=I_{0 m}$.

The model parameters are: the recruitment rate of human population $\Lambda_{h}$, the recruitment rate of mosquito population $\Lambda_{m}$, the effective contact rate human to human $\beta_{1}$, the effective contact rate mosquitoes to human $\beta_{2}$, the effective contact rate human to mosquitoes $\mu$, the natural death rate of human $k_{1}$, the natural death rate of mosquitoes $k_{2}$.

Model (1) does not include the internal memory effects of the system. To improve the model, we change the first-order time derivative to the Caputo fractional derivative of order $v$. With this change, the right- and left-hand sides will not have the same dimension. To solve this problem, we use an auxiliary parameter $\theta$, having the dimension of sec., to change the fractional operator so that the sides have the same dimension $([47,48])$. According to the explanation presented, the transmission model of Zika virus for $t \geq 0$ and 
$v \in(0,1)$ is given as follows:

$$
\left\{\begin{array}{l}
\theta^{v-1 C} D_{t}^{v} S_{h}(t)=\Lambda_{h}-\beta_{1} S_{h} I_{h}-\beta_{2} S_{h} I_{m}-k_{1} S_{h}, \\
\theta^{v-1 C} D_{t}^{v} I_{h}(t)=\beta_{1} S_{h} I_{h}+\beta_{2} S_{h} I_{m}-k_{1} I_{h}, \\
\theta^{v-1 C} D_{t}^{v} S_{m}(t)=\Lambda_{m}-\mu S_{m} I_{h}-k_{2} S_{m}, \\
\theta^{v-1 C} D_{t}^{v} I_{m}(t)=\mu S_{m} I_{h}-k_{2} I_{m},
\end{array}\right.
$$

where the initial conditions are $S_{h}(0)=S_{0 h}, I_{h}(0)=I_{0 h}, S_{m}(0)=S_{0 m}, I_{m}(0)=I_{0 m}$.

\subsection{Nonnegative solution}

Consider $\Phi=\left\{\left(S_{h}, I_{h}, S_{m}, I_{m}\right) \in R_{4}^{+}: N_{h}(t) \leq \frac{\Lambda_{h}}{k_{1}}, N_{m}(t) \leq \frac{\Lambda_{m}}{k_{2}}\right\}$, we show that the closed set $\Phi$ is the region of the feasibility of system (2).

Lemma 3.1 The closed set $\Phi$ is positively invariant with respect to fractional system (2).

Proof To obtain the fractional derivative of the total population, we add the first two relations in system (2). So

$$
\theta^{v-1 C} D_{t}^{v} N_{h}(t)=\Lambda_{h}-k_{1} N_{h}(t)
$$

where $N_{h}(t)=S_{h}(t)+I_{h}(t)$. Using the Laplace transform, we obtain

$$
N_{h}(t)=N_{h}(0) E_{v}\left(-k_{1} \theta^{1-v} t^{\nu}\right)+\int_{0}^{t} \Lambda_{h} \theta^{1-v} \eta^{\nu-1} E_{v, v}\left(-k_{1} \theta^{1-\eta} \eta^{v}\right) d \eta
$$

where $N_{h}(0)$ is the initial human population size, and the terms $E_{v}, E_{v, v}$ in the above equation are represented by the Mittag-Leffler function and its general form defined by

$$
E_{v}(z)=\sum_{j=0}^{\infty} \frac{z^{j}}{\Gamma(1+j v)}, \quad E_{v, v}=\sum_{j=0}^{\infty} \frac{z^{j}}{\Gamma(v+j v)}, \quad v>0 .
$$

With some calculations, we get

$$
\begin{aligned}
N_{h}(t) & =N_{h}(0) E_{v}\left(-k_{1} \theta^{1-v} t^{\nu}\right)+\int_{0}^{t} \Lambda_{h} \theta^{1-\nu} \eta^{\nu-1} \sum_{i=0}^{\infty} \frac{(-1)^{i} k_{1}^{i} \theta^{i(1-v)} \eta^{i v}}{\Gamma(i v+v)} d \eta \\
& =\frac{\Lambda_{h} \theta^{1-\nu}}{k_{1} \theta^{1-\nu}}+E_{\nu}\left(-k_{1} \theta^{1-v} t^{\nu}\right)\left(N_{h}(0)-\frac{\Lambda_{h} \theta^{1-\nu}}{k_{1} \theta^{1-\nu}}\right) \\
& =\frac{\Lambda_{h}}{k_{1}}+E_{\nu}\left(-k_{1} \theta^{1-v} t^{\nu}\right)\left(N_{h}(0)-\frac{\Lambda_{h}}{k_{1}}\right) .
\end{aligned}
$$

Thus, if $N_{h}(0) \leq \frac{\Lambda_{h}}{k_{1}}$, then for $t>0, N_{h}(t) \leq \frac{\Lambda_{h}}{k_{1}}$. Similarly, we can prove for $N_{m}$ that if $N_{m}(0) \leq \frac{\Lambda_{m}}{k_{2}}$, then for $t>0, N_{m}(t) \leq \frac{\Lambda_{m}}{k_{2}}$. Consequently, the closed set $\Phi$ is positively invariant with respect to fractional model (2). 


\subsection{Equilibrium points and reproduction number}

To determine the equilibrium points of fractional order system (2), we solve the following equations:

$$
{ }^{C} D^{v} S_{h}(t)={ }^{C} D^{v} I_{h}(t)={ }^{C} D^{v} S_{m}(t)={ }^{C} D^{v} I_{m}(t)=0 .
$$

By solving the above algebraic equations, we obtain two equilibrium points of system (2). The disease-free equilibrium point is obtained as $E^{0}=\left(\frac{\Lambda_{h}}{k_{1}}, 0, \frac{\Lambda_{m}}{k_{2}}\right)$. In addition, if $R_{0}>1$, then system (2) has a positive endemic equilibrium point $E^{*}=\left(S_{h}^{*}, I_{h}^{*}, S_{m}^{*}, I_{m}^{*}\right)$, where

$$
\begin{aligned}
S_{h}^{*} & =\frac{k_{2} k_{1}}{\left(\beta_{2} \mu S_{m}^{*}+k_{2} \beta_{1}\right)}, \\
I_{h}^{*} & =\frac{\Lambda_{h} \beta_{2} \mu S_{m}^{*}+\Lambda_{h} k_{2} \beta_{1}-k_{2} k_{1}^{2}}{k_{1}\left(\beta_{2} \mu S_{m}^{*}+k_{2} \beta_{1}\right)}, \\
I_{m}^{*} & =\frac{\mu\left(\Lambda_{h} \beta_{2} \mu S_{m}^{*}+\Lambda_{h} k_{2} \beta_{1}-k_{2} k_{1}^{2}\right) S_{m}^{*}}{k_{1}\left(\beta_{2} \mu S_{m}^{*}+k_{2} \beta_{1}\right) k_{2}} .
\end{aligned}
$$

Also, $R_{0}$ is the basic reproduction number and is obtained using the next generation method [49]. To find $R_{0}$, we first consider the system as follows:

$$
{ }^{C} D^{v} \Psi(t)=F(\Psi(t))-V(\Psi(t))
$$

where

$$
F(\Psi(t))=\theta^{1-\nu}\left[\begin{array}{c}
\beta_{1} S_{h} I_{h}+\beta_{2} S_{h} I_{m} \\
\mu S_{m} I_{h}
\end{array}\right]
$$

and

$$
V(\Psi(t))=\theta^{1-v}\left[\begin{array}{l}
k_{1} I_{h} \\
k_{2} I_{m}
\end{array}\right] .
$$

At $E^{0}$, the Jacobian matrix for $F$ and $V$ is obtained as follows:

$$
J_{F}\left(E_{0}\right)=\theta^{1-v}\left[\begin{array}{cc}
\frac{\beta_{1} \Lambda_{h}}{k_{1}} & \frac{\beta_{2} \Lambda_{h}}{k_{2}} \\
\frac{\mu \Lambda_{m}}{k_{2}} & 0
\end{array}\right], \quad J_{v}\left(E_{0}\right)=\theta^{1-\nu}\left[\begin{array}{cc}
k_{1} & 0 \\
0 & k_{2}
\end{array}\right] .
$$

$F V^{-1}$ is the next generation matrix for the system (2), and the basic reproduction number is obtained from $R_{0}=\rho\left(F V^{-1}\right)$, where $\rho\left(F V^{-1}\right)$ is the eigenvalue of matrix $F V^{-1}$. We get

$$
R_{0}=\frac{\beta_{1} k_{2} \Lambda_{h}+\sqrt{\beta_{1}^{2} \Lambda_{h}^{2} k_{2}^{2}+4 k_{1}^{2} \beta_{2} \mu \Lambda_{h} \Lambda_{m}}}{2 k_{2} k_{1}^{2}} .
$$

This basic reproduction number $R_{0}$ is an epidemiologic metric used to describe the contagiousness or transmissibility of infectious agents. 


\subsection{Stability of equilibrium point}

To investigate the stability of the equilibrium point, we first consider the Jacobian matrix of system (2) as follows:

$$
J=\theta^{1-\nu}\left[\begin{array}{cccc}
-\beta_{1} I_{h}-\beta_{2} I_{m}-k_{1} & -\beta_{1} S_{h} & 0 & -\beta_{2} S_{h} \\
\beta_{1} I_{h}+\beta_{2} I_{m} & \beta_{1} S_{h}-k_{1} & 0 & \beta_{2} S_{h} \\
0 & -\mu S_{m} & -\mu I_{h}-k_{2} & 0 \\
0 & \mu S_{m} & \mu I_{h} & -k_{2}
\end{array}\right] .
$$

At $E_{0}$, the Jacobian matrix of system (2) is

$$
J=\theta^{1-\nu}\left[\begin{array}{cccc}
-k_{1} & -\beta_{1} \frac{\Lambda_{h}}{k_{1}} & 0 & -\beta_{2} \frac{\Lambda_{h}}{k_{1}} \\
0 & \beta_{1} \frac{\Lambda_{h}}{k_{1}}-k_{1} & 0 & \beta_{2} \frac{\Lambda_{h}}{k_{1}} \\
0 & -\mu \frac{\Lambda_{m}}{k_{2}} & -k_{2} & 0 \\
0 & \mu \frac{\Lambda_{m}}{k_{2}} & 0 & -k_{2}
\end{array}\right]
$$

Theorem 3.2 If $R_{0}<1$, the equilibrium point $E^{0}$ of system (2) is locally asymptotically stable.

Proof At the disease-free equilibrium point $E^{0}$, the characteristic equation of the Jacobian matrix is $\operatorname{det}\left(\lambda I-J\left(E^{0}\right)\right)=0$. Then we obtain

$$
\theta^{1-v}\left(\lambda+k_{1}\right)\left(\lambda+k_{2}\right)\left(\lambda^{2}+B \lambda+C\right)=0,
$$

where $B=k_{1}+k_{2}-\frac{\beta_{1} \Lambda_{h}}{k_{1}}$ and $C=2 k_{1} k_{2}-\frac{k_{2} \beta_{1} \Lambda_{h}}{k_{1}}-\frac{\mu \beta_{2} \Lambda_{m} \Lambda_{h}}{k_{1} k_{2}}$. By simplifying the above equations, the eigenvalues of characteristic equation are obtained as $\lambda_{1}=-k_{1}, \lambda_{2}=-k_{2}$ and the roots of the equation

$$
\lambda^{2}+B \lambda+C=0
$$

If $R_{0}<1$, since all of the parameters are positive, then

$$
\frac{\beta_{1} k_{2} \Lambda_{h}+\sqrt{\beta_{1}^{2} \Lambda_{h}^{2} k_{2}^{2}}}{2 k_{2} k_{1}^{2}}<1 \quad \Rightarrow \quad \frac{\beta_{1} \Lambda_{h}}{k_{1}}<k_{1} \Rightarrow B=k_{1}+k_{2}-\frac{\beta_{1} \Lambda_{h}}{k_{1}}>k_{2}>0 .
$$

Also, from $R_{0}<1$ we have

$$
\begin{aligned}
& \frac{\sqrt{4 k_{1}^{2} \beta_{2} \mu \Lambda_{h} \Lambda_{m}}}{2 k_{2} k_{1}^{2}}<1 \\
& \Rightarrow \quad \frac{\beta_{2} \mu \Lambda_{m} \Lambda_{h}}{k_{1} k_{2}}<k_{1} k_{2}, \\
& \Rightarrow \quad 2 k_{1} k_{2}-\frac{k_{2} \beta_{1} \Lambda_{h}}{k_{1}}-\frac{\mu \beta_{2} \Lambda_{m} \Lambda_{h}}{k_{1} k_{2}}>2 k_{1} k_{2}-k_{1} k_{2}-k_{1} k_{2} \Rightarrow C>0 .
\end{aligned}
$$

Since $B>0, C>0$, applying the Routh-Hurwitz criteria, we obtain that $E_{0}$ is locally asymptotically stable. 


\section{Existence and uniqueness of solution}

To show that the system has a unique solution, we write system (2) as follows:

$$
\left\{\begin{array}{l}
\theta^{v-1 C} D_{t}^{v} S_{h}(t)=W_{1}\left(t, S_{h}(t)\right) \\
\theta^{v-1 C} D_{t}^{v} I_{h}(t)=W_{2}\left(t, I_{h}(t)\right) \\
\theta^{v-1 C} D_{t}^{v} S_{m}(t)=W_{3}\left(t, S_{m}(t)\right) \\
\theta^{v-1 C} D_{t}^{v} I_{m}(t)=W_{4}\left(t, I_{m}(t)\right)
\end{array}\right.
$$

By applying integral on both sides of the above equations, we have

$$
\left\{\begin{array}{l}
S_{h}(t)-S_{h}(0)=\frac{\theta^{1-v}}{\Gamma(v)} \int_{0}^{t} W_{1}\left(\tau, S_{h}\right)(t-\tau)^{\nu-1} d \tau, \\
I_{h}(t)-I_{h}(0)=\frac{\theta^{1-v}}{\Gamma(v)} \int_{0}^{t} W_{2}\left(\tau, I_{h}\right)(t-\tau)^{\nu-1} d \tau, \\
S_{m}(t)-S_{m}(0)=\frac{\theta^{1-v}}{\Gamma(v)} \int_{0}^{t} W_{3}\left(\tau, S_{m}\right)(t-\tau)^{v-1} d \tau, \\
I_{m}(t)-I_{m}(0)=\frac{\theta^{1-v}}{\Gamma(v)} \int_{0}^{t} W_{4}\left(\tau, I_{m}\right)(t-\tau)^{v-1} d \tau .
\end{array}\right.
$$

We show that the kernels $W_{i}, i=1,2,3,4$, satisfy the Lipschitz condition and contraction.

Theorem 4.1 The kernel $W_{1}$ satisfies the Lipschitz condition and contraction if the following inequality holds:

$$
0 \leq \beta_{1} u_{1}+\beta_{2} u_{2}+k_{1}<1
$$

Proof For $S_{h}$ and $S_{1 h}$, we have

$$
\begin{aligned}
\left\|W_{1}\left(t, S_{h}\right)-W_{1}\left(t, S_{1 h}\right)\right\| & =\left\|\beta_{1} I_{h}\left(S_{h}-S_{1 h}\right)+\beta_{2} I_{m}\left(S_{h}-S_{1 h}\right)+k_{1}\left(S_{h}-S_{1 h}\right)\right\|, \\
& \leq \beta_{1}\left\|I_{h}\right\|\left\|S_{h}-S_{1 h}\right\|+\beta_{2}\left\|I_{m}\right\|\left\|S_{h}-S_{1 h}\right\|+k_{1}\left\|S_{h}-S_{1 h}\right\|, \\
& \leq\left(\beta_{1}\left\|I_{h}\right\|+\beta_{2}\left\|I_{m}\right\|+k_{1}\right)\left\|S_{h}-S_{1 h}\right\|, \\
& \leq\left(\beta_{1} u_{1}+\beta_{2} u_{2}+k_{1}\right)\left\|S_{h}-S_{1 h}\right\| .
\end{aligned}
$$

Suppose that $M_{1}=\beta_{1} u_{1}+\beta_{2} u_{2}+k_{1}$, where $\left\|I_{h}(t)\right\| \leq u_{1},\left\|I_{m}\right\| \leq u_{2}$ are bounded functions, then

$$
\left\|W_{1}\left(t, S_{h}\right)-W_{1}\left(t, S_{1 h}\right)\right\| \leq M_{1}\left\|S_{h}(t)-S_{1 h}(t)\right\| .
$$

Thus, for $W_{1}$, the Lipschitz condition is obtained, and if $0 \leq \beta_{1} u_{1}+\beta_{2} u_{2}+k_{1}<1$ then $W_{1}$ is a contraction.

Similarly, we can prove that $W_{i}, i=2,3,4$, satisfies the Lipschitz condition as follows:

$$
\left\{\begin{array}{l}
\left\|W_{2}\left(t, I_{h}\right)-W_{2}\left(t, I_{1 h}\right)\right\| \leq M_{2}\left\|I_{h}(t)-I_{1 h}(t)\right\|, \\
\left\|W_{3}\left(t, S_{m}\right)-W_{3}\left(t, S_{1 m}\right)\right\| \leq M_{3}\left\|S_{m}(t)-S_{1 m}(t)\right\|, \\
\left\|W_{4}\left(t, I_{m}\right)-W_{4}\left(t, I_{1 m}\right)\right\| \leq M_{4}\left\|I_{m}(t)-I_{1 m}(t)\right\|,
\end{array}\right.
$$

where $\left\|S_{h}(t)\right\| \leq u_{3},\left\|S_{m}(t)\right\| \leq u_{4}$, and $M_{2}=\beta_{1} u_{3}+k_{1}, M_{3}=\mu u_{1}+k_{2}, M_{4}=k_{2}$ are bounded functions, if $0 \leq M_{i}<1, i=2,3,4$, then $W_{i}, i=2,3,4$, are contraction. 
According to system (3), consider the following recursive forms:

$$
\begin{aligned}
& \Phi_{1 n}(t)=S_{n h}(t)-S_{(n-1) h}(t)=\frac{\theta^{1-v}}{\Gamma(v)} \int_{0}^{t}\left(W_{1}\left(\tau, S_{(n-1) h}\right)-W_{1}\left(\tau, S_{(n-2) h}\right)\right)(t-\tau)^{\nu-1} d \tau, \\
& \Phi_{2 n}(t)=I_{n h}(t)-I_{(n-1) h}(t)=\frac{\theta^{1-v}}{\Gamma(v)} \int_{0}^{t}\left(W_{2}\left(\tau, I_{(n-1) h}\right)-W_{2}\left(\tau, I_{(n-2) h}\right)\right)(t-\tau)^{\nu-1} d \tau, \\
& \Phi_{3 n}(t)=S_{n h}(t)-S_{(n-1) h}(t)=\frac{\theta^{1-v}}{\Gamma(v)} \int_{0}^{t}\left(W_{3}\left(\tau, S_{(n-1) h}\right)-W_{3}\left(\tau, S_{(n-2) h}\right)\right)(t-\tau)^{\nu-1} d \tau, \\
& \Phi_{4 n}(t)=I_{n h}(t)-I_{(n-1) h}(t)=\frac{\theta^{1-v}}{\Gamma(v)} \int_{0}^{t}\left(W_{4}\left(\tau, I_{(n-1) h}\right)-W_{4}\left(\tau, I_{(n-2) h}\right)\right)(t-\tau)^{\nu-1} d \tau,
\end{aligned}
$$

with the initial conditions $S_{0 h}(t)=S_{h}(0), I_{0 h}(t)=I_{h}(0), S_{0 m}(t)=S_{m}(0)$, and $I_{0 m}(t)=I_{m}(0)$. We take the norm of the first equation in the above system, then

$$
\begin{aligned}
\left\|\Phi_{1 n}(t)\right\| & =\left\|S_{n h}(t)-S_{(n-1) h}(t)\right\| \\
& =\left\|\frac{\theta^{1-v}}{\Gamma(v)} \int_{0}^{t}\left(W_{1}\left(\tau, S_{(n-1) h}\right)-W_{1}\left(\tau, S_{(n-2) h}\right)\right)(t-\tau)^{\nu-1} d \tau\right\| \\
& \left.\leq \frac{\theta^{1-v}}{\Gamma(\nu)} \int_{0}^{t} \| W_{1}\left(\tau, S_{(n-1) h}\right)-W_{1}\left(\tau, S_{(n-2) h}\right)\right)(t-\tau)^{\nu-1} \| d \tau .
\end{aligned}
$$

By Lipschitz condition (4), we have

$$
\left\|\Phi_{1 n}(t)\right\| \leq \frac{\theta^{1-v}}{\Gamma(v)} M_{1} \int_{0}^{t}\left\|\Phi_{1(n-1)}(\tau)\right\| d \tau .
$$

In a similar way, we obtained

$$
\begin{aligned}
\left\|\Phi_{2 n}(t)\right\| & \leq \frac{\theta^{1-v}}{\Gamma(v)} M_{2} \int_{0}^{t}\left\|\Phi_{2(n-1)}(\tau)\right\| d \tau, \\
\left\|\Phi_{3 n}(t)\right\| & \leq \frac{\theta^{1-v}}{\Gamma(v)} M_{3} \int_{0}^{t}\left\|\Phi_{3(n-1)}(\tau)\right\| d \tau, \\
\left\|\Phi_{4 n}(t)\right\| & \leq \frac{\theta^{1-v}}{\Gamma(v)} M_{4} \int_{0}^{t}\left\|\Phi_{4(n-1)}(\tau)\right\| d \tau .
\end{aligned}
$$

Then we can obtain

$$
\begin{array}{ll}
S_{n h}(t)=\sum_{i=1}^{n} \Phi_{1 i}(t), & I_{n h}(t)=\sum_{i=1}^{n} \Phi_{2 i}(t), \\
S_{n m}(t)=\sum_{i=1}^{n} \Phi_{3 i}(t), & I_{n m}(t)=\sum_{i=1}^{n} \Phi_{4 i}(t) .
\end{array}
$$

We prove the existence of a solution in the next theorem.

Theorem 4.2 The fractional model of Zika virus (2) has a solution if there exists $t_{1}$ such that

$$
\frac{\theta^{1-v}}{\Gamma(v)} t_{1} M_{i}<1
$$


Proof From the recursive technique and Eq. (5) and Eq. (6), we conclude that

$$
\begin{aligned}
\left\|\Phi_{1 n}(t)\right\| & \leq\left\|S_{n h}(0)\right\|\left[\frac{\theta^{1-v}}{\Gamma(v)} M_{1} t\right]^{n}, \\
\left\|\Phi_{2 n}(t)\right\| & \leq\left\|I_{n h}(0)\right\|\left[\frac{\theta^{1-v}}{\Gamma(v)} M_{2} t\right]^{n}, \\
\left\|\Phi_{3 n}(t)\right\| & \leq\left\|S_{n m}(0)\right\|\left[\frac{\theta^{1-v}}{\Gamma(v)} M_{3} t\right]^{n}, \\
\left\|\Phi_{4 n}(t)\right\| & \leq\left\|I_{n m}(0)\right\|\left[\frac{\theta^{1-v}}{\Gamma(v)} M_{4} t\right]^{n} .
\end{aligned}
$$

Then the system has a solution, and also it is continuous. Now we show that the above functions construct a solution for model (2). We assume that

$$
\begin{aligned}
& S_{h}(t)-S_{h}(0)=S_{n h}(t)-\mathrm{B}_{1 n}(t), \\
& I_{h}(t)-I_{h}(0)=I_{n h}(t)-\mathrm{B}_{2 n}(t), \\
& S_{m}(t)-S_{m}(0)=S_{n m}(t)-\mathrm{B}_{3 n}(t), \\
& I_{m}(t)-I_{m}(0)=I_{n m}(t)-\mathrm{B}_{4 n}(t) .
\end{aligned}
$$

Thus

$$
\begin{aligned}
\left\|\mathrm{B}_{1 n}(t)\right\| & =\left\|\frac{\theta^{1-v}}{\Gamma(v)} \int_{0}^{t}\left(W_{1}\left(\tau, S_{h}\right)-W_{1}\left(\tau, S_{(n-1) h}\right)\right) d \tau\right\| \\
& \leq \frac{\theta^{1-v}}{\Gamma(v)} \int_{0}^{t}\left\|W_{1}\left(\tau, S_{h}\right)-W_{1}\left(\tau, S_{(n-1) h}\right)\right\| d \tau \\
& \leq \frac{\theta^{1-v}}{\Gamma(v)} M_{1}\left\|S_{h}-S_{(n-1) h}\right\| t .
\end{aligned}
$$

By repeating the method, we obtain

$$
\left\|\mathrm{B}_{1 n}(t)\right\| \leq\left[\frac{\theta^{1-v}}{\Gamma(v)} t\right]^{n+1} M_{1}^{n+1} h
$$

At $t_{1}$, we get

$$
\left\|\mathrm{B}_{1 n}(t)\right\| \leq\left[\frac{\theta^{1-v}}{\Gamma(v)} t_{1}\right]^{n+1} M_{1}^{n+1} h
$$

Taking limit on recent equation as $n$ approaches $\infty$, we obtain $\left\|\mathrm{B}_{1 n}(t)\right\| \rightarrow 0$. In the same way, we can show that $\left\|\mathrm{B}_{i n}(t)\right\| \rightarrow 0, i=2,3,4$. This completes the proof.

In the following, we show that system (2) has a unique solution. We suppose that the system has another solution such as $S_{1 h}(t), I_{1 h}(t), S_{1 m}(t)$, and $I_{1 m}(t)$, then we have

$$
S_{h}(t)-S_{1 h}(t)=\frac{\theta^{1-v}}{\Gamma(v)} \int_{0}^{t}\left(W_{1}\left(\tau, S_{h}\right)-W_{1}\left(\tau, S_{1 h}\right)\right) d \tau
$$


By taking the norm from this equation, we obtain

$$
\left\|S_{h}(t)-S_{1 h}(t)\right\|=\frac{\theta^{1-\nu}}{\Gamma(\nu)} \int_{0}^{t}\left\|W_{1}\left(\tau, S_{h}\right)-W_{1}\left(\tau, S_{1 h}\right)\right\| d \tau .
$$

It follows from Lipschitz condition (4) that

$$
\left\|S_{h}(t)-S_{1 h}(t)\right\| \leq \frac{\theta^{1-v}}{\Gamma(v)} M_{1} t\left\|S_{h}(t)-S_{1 h}(t)\right\| .
$$

Then

$$
\left\|S_{h}(t)-S_{1 h}(t)\right\|\left(1-\frac{\theta^{1-v}}{\Gamma(v)} M_{1} t\right) \leq 0
$$

Theorem 4.3 The solution of the transmission model of Zika virus is unique if the following condition holds:

$$
1-\frac{\theta^{1-v}}{\Gamma(v)} M_{1} t>0 .
$$

Proof Suppose that condition (7) holds

$$
\left\|S_{h}(t)-S_{1 h}(t)\right\|\left(1-\frac{\theta^{1-v}}{\Gamma(v)} M_{1} t\right) \leq 0 .
$$

Then $\left\|S_{h}(t)-S_{1 h}(t)\right\|=0$. So, we obtain $S_{h}(t)=S_{1 h}(t)$. Similarly, we can show the same equality for $I_{h}, S_{m}, I_{m}$.

\section{Numerical results}

Using the fractional Euler method for Caputo derivative, we present the approximate solutions for the transmission model of Zika virus [50]. We present simulations for investigating the dynamics of the system.

\subsection{Numerical method}

We consider system (2) in the compact form as follows:

$$
\theta^{v-1 C} D_{t}^{v} u(t)=p(t, u(t)), \quad u(0)=u_{0}, 0 \leq t \leq T<\infty,
$$

where $u=\left(S_{h}, I_{h}, S_{m}, I_{m}\right) \in R_{+}^{4}, u_{0}=\left(S_{0 h}, I_{0 h}, S_{0 m}, I_{0 m}\right)$ is the initial vector, and $p(t) \in R$ is a continuous vector function satisfying the Lipschitz condition

$$
\left\|p\left(u_{1}(t)\right)-p\left(u_{2}(t)\right)\right\| \leq r\left\|u_{1}(t)-u_{2}(t)\right\|, \quad r>0 .
$$

Applying the fractional integral operator corresponding Caputo derivative to equation (8), we obtain

$$
u(t)=\theta^{1-v}\left[u_{0}+I^{v} p(u(t))\right], \quad 0 \leq t \leq T<\infty .
$$


Set $q=\frac{T-0}{N}$ and $t_{n}=n q$, where $t \in[0, T]$ and $N$ is a natural number and $n=0,1,2, \ldots, N$. Let $u_{n}$ be the approximation of $u(t)$ at $t=t_{n}$. Using the fractional Euler method [50], we get

$$
u_{n+1}=\theta^{1-v}\left[u_{0}+\frac{q^{v}}{\Gamma(v+1)} \sum_{j=0}^{n} z_{n+1, j} p\left(t_{j}, u_{j}\right)\right], \quad j=0,1,2, \ldots, N-1
$$

where

$$
z_{n+1, j}=(n+1-j)^{\nu}-(n-j)^{\nu}, \quad j=0,1,2, \ldots, n .
$$

The stability analysis of the obtained scheme has been proved in Theorem (3.1) in [50].

Thus, the solution of system (2) is written as follows:

$$
\begin{aligned}
& S_{(n+1) h}=\theta^{1-v}\left[S_{0 h}+\frac{q^{v}}{\Gamma(v+1)} \sum_{j=0}^{n} z_{n+1, j} g_{1}\left(t_{j}, u_{j}\right)\right], \\
& I_{(n+1) h}=\theta^{1-v}\left[I_{0 h}+\frac{q^{v}}{\Gamma(v+1)} \sum_{j=0}^{n} z_{n+1, j} g_{2}\left(t_{j}, u_{j}\right)\right], \\
& S_{(n+1) m}=\theta^{1-v}\left[S_{0 m}+\frac{q^{v}}{\Gamma(v+1)} \sum_{j=0}^{n} z_{n+1, j} g_{3}\left(t_{j}, u_{j}\right)\right], \\
& I_{(n+1) m}=\theta^{1-v}\left[I_{0 m}+\frac{q^{v}}{\Gamma(v+1)} \sum_{j=0}^{n} z_{n+1, j} g_{4}\left(t_{j}, u_{j}\right)\right],
\end{aligned}
$$

where $z_{n+1, j}=(n+1-j)^{v}-(n-j)^{v}, g_{1}(t, u(t))=\Lambda_{h}-\beta_{1} S_{h}(t) I_{h}(t)-\beta_{2} I_{m}(t)(t) S_{h}(t)-k_{1} S_{h}(t)$, $g_{2}(t, u(t))=\beta_{1} S_{h}(t) I_{h}(t)+\beta_{2} I_{m}(t)(t) S_{h}(t)-k_{1} I_{h}(t), g_{3}(t, u(t))=\Lambda_{m}-\mu S_{m}(t) I_{h}(t)-k_{2} S_{m}(t)$, $g_{4}(t, u(t))=\mu S_{m}(t) I_{h}(t)-k_{2} I_{m}(t)$.

\subsection{Simulation}

In this section, using numerical results, we investigate the behavior of the answers of the transmission model of Zika virus obtained from system (2). The numerical values of the model parameters are considered as $\Lambda_{h}=1.2, \Lambda_{m}=0.3, k_{1}=0.004, k_{2}=0.0014$, $\beta_{1}=0.125 \times 10^{-4}, \beta_{2}=0.4 \times 10^{-4}, \mu=0.475 \times 10^{-5}$, and we take its modification parameter as $\theta=0.99$. Also, the initial values are considered as $S_{h}(0)=800, I_{h}(0)=200, S_{m}=600$, $I_{m}=300$.

Figure 1 shows susceptible people $S_{h}$ and Fig. 2 shows infected people $I_{h}$ for the integerorder derivative $v=1$ and fractional-order derivative $v=0.98,0.96,0.94,0.92, .09$. As Fig. 1 shows, the behavior of $S_{h}$ in both types of integer-order and fractional-order derivative is the same and decreasing, that is, over time, all healthy people are exposed to the disease, but the obtained numerical values are different, and as the derivative order decreases, the resulting numerical value increases.

In Fig. 2, you can see that the behavior of $I_{h}$ is the same in both derivatives, and the resulting numerical values are different. As the derivative order decreases, the resulting numerical value for $I_{h}$ increases, and this difference in the obtained value is significant 


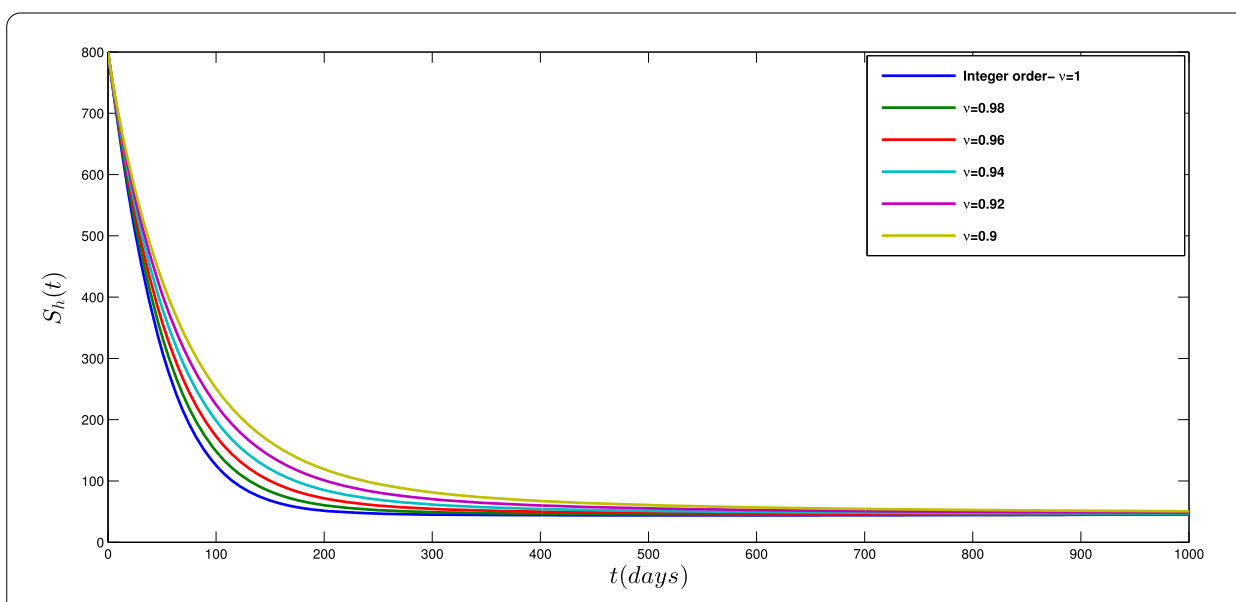

Figure 1 Plots of susceptible people $S_{h}(t)$ for integer-order derivative at $v=1$ and fractional-order derivative with various values of $v=0.98,0.96,0.94,0.92,0.9$

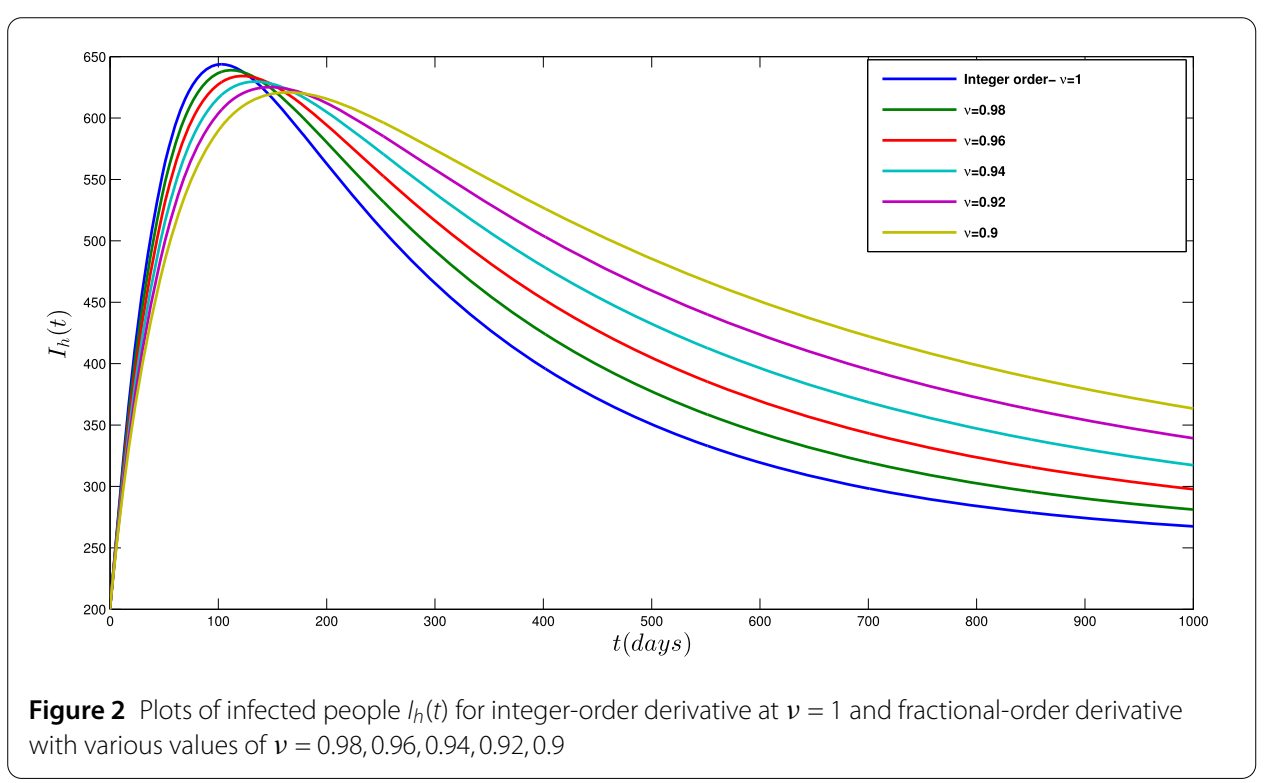

over time. Figure 2 also shows that $I_{h}$ passes the peak in the first 100 days and the number of infected people gradually decreases and tends to the equilibrium point.

Figures 3 and 4 show susceptible mosquitoes $S_{m}$ and infected mosquitoes $I_{m}$, respectively. In these diagrams, you can see that the behavior of the functions is the same in both derivatives and the resulting numerical values are different. These figures also show that over time the population of healthy mosquitoes decreases and they are more exposed to the disease, while the number of infected mosquitoes increases.

\section{Conclusion}

In this paper, a mathematical model for the transmission of Zika virus between humans and mosquitoes is presented using the Caputo fractional-order derivative. The region of the feasibility of system (2), the equilibrium points, and the reproduction number have been determined, and the stability of the equilibrium point $E^{0}$ has been checked. Using a fixed point theory, the existence of a unique solution for model (2) has been proven. In 


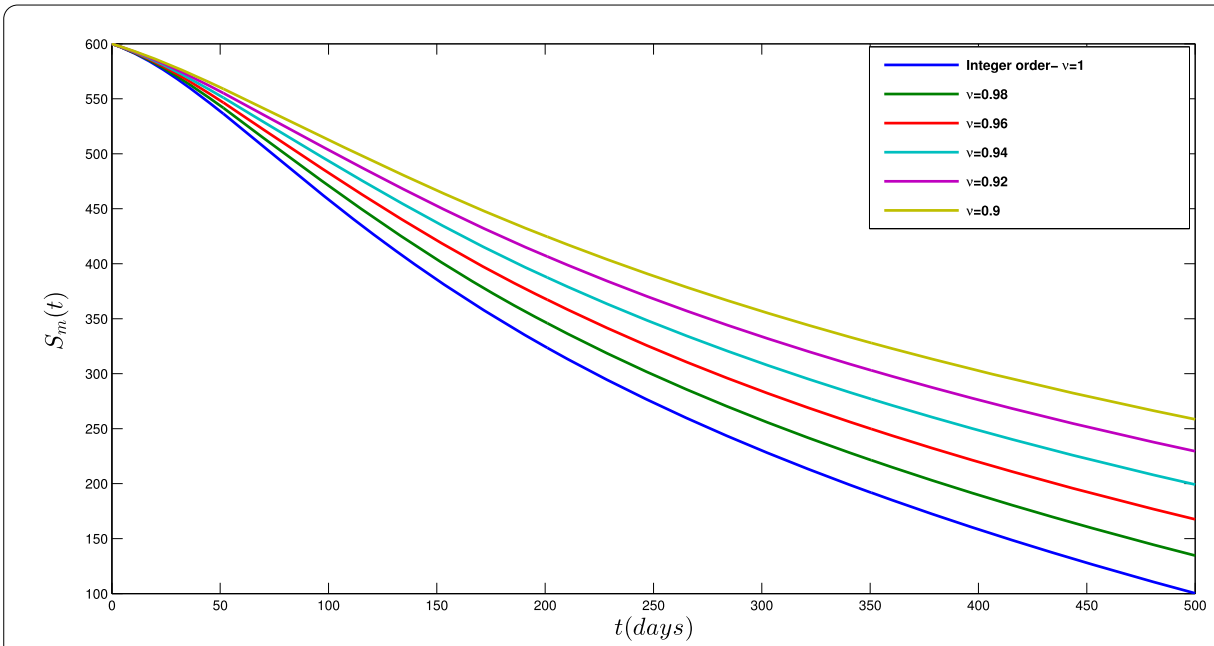

Figure 3 Plots of $S_{m}(t)$ for integer-order derivative at $v=1$ and fractional-order derivative with various values of $v=0.98,0.96,0.94,0.92,0.9$

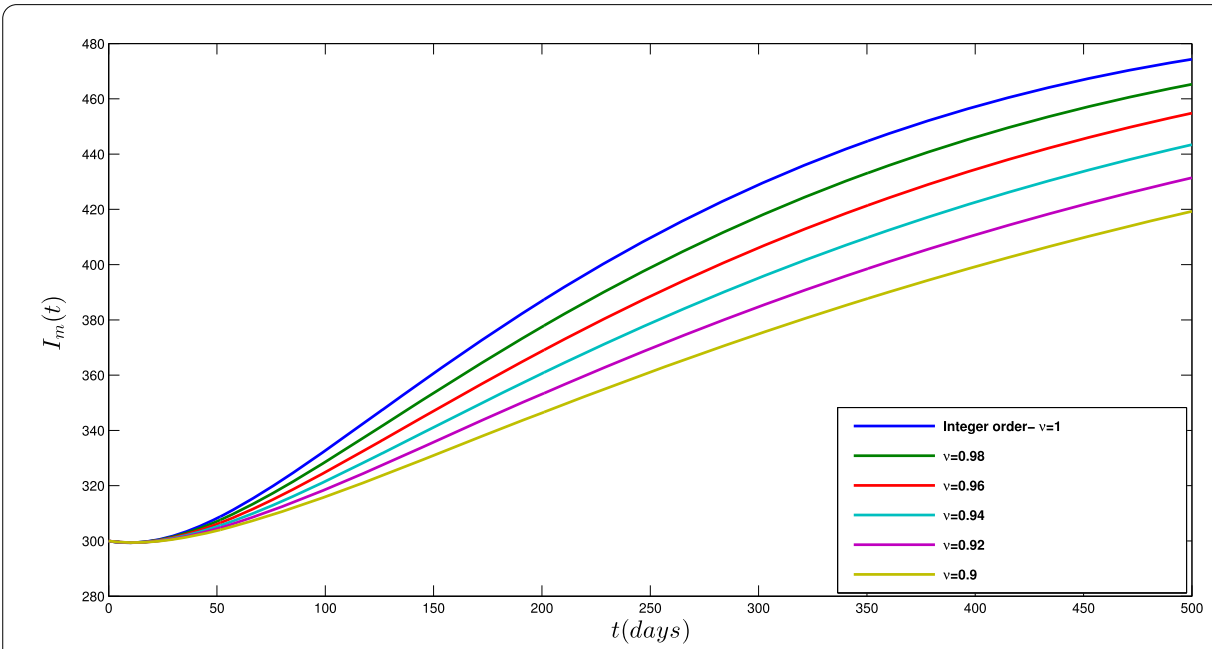

Figure 4 Plots of $/ \mathrm{m}(t)$ for integer-order derivative at $v=1$ and fractional-order derivative with various values of $v=0.98,0.96,0.94,0.92,0.9$

the numerical section, the answers of system (2) are calculated using the Euler method, and the results are compared for the integer-order model and the fractional-order model in numerical results. The results show that the behavior of the obtained functions in both types of derivatives is the same, but the resulting numerical values are different, especially the difference in values increases over time.

\section{Acknowledgements}

The first author was supported by Miandoab Branch, Islamic Azad University. The second author was supported by Azarbaijan Shahid Madani University. The third author was supported by Bojnord University. The authors express their gratitude to dear unknown referees for their helpful suggestions which improved the final version of this paper. 
Availability of data and materials

Data sharing not applicable to this article as no datasets were generated or analyzed during the current study.

\section{Ethics approval and consent to participate}

Not applicable.

\section{Competing interests}

The authors declare that they have no competing interests.

\section{Consent for publication}

Not applicable.

\section{Authors' contributions}

The authors declare that the study was realized in collaboration with equal responsibility. All authors read and approved the final manuscript.

\section{Author details}

${ }^{1}$ Institute of Research and Development, Duy Tan University, Da Nang 550000, Vietnam. ${ }^{2}$ Faculty of Natural Sciences, Duy Tan University, Da Nang 550000, Vietnam. ${ }^{3}$ Department of Medical Research, China Medical University Hospital, China Medical University, Taichung, Taiwan. ${ }^{4}$ Department of Mathematics, Miandoab Branch, Islamic Azad University, Miandoab, Iran. ${ }^{5}$ Department of Electrical Engineering, University of Bojnord, P.O. Box, 94531-1339, Bojnord, Iran. ${ }^{6}$ Department of Mathematics, Near East University TRNC, Mersin 10, Turkey.

\section{Publisher's Note}

Springer Nature remains neutral with regard to jurisdictional claims in published maps and institutional affiliations.

Received: 2 July 2020 Accepted: 8 October 2020 Published online: 20 October 2020

\section{References}

1. Chen, T.M., Rui, J., Wang, Q.P., Zhao, Z.Y., Cui, J.A., Yin, L.: A mathematical model for simulating the phase-based transmissibility of a novel coronavirus. Infect. Dis. Poverty 9, 24 (2020). https://doi.org/10.1186/s40249-020-00640-3

2. Dighe, A., Jombart, T., Van Kerkhove, M., Ferguson, N.: A mathematical model of the transmission of middle east respiratory syndrome coronavirus in dromedary camels (Camelus dromedarius). Int. J. Infect. Dis. 79(S1), 03.002 (2019). https://doi.org/10.1016/j.ijid.2018.11.023

3. Tan, X., Yuan, L., Zhou, J., Zheng, Y., Yang, F.: Modeling the initial transmission dynamics of influenza A H1N1 in Guangdong province, China. Int. J. Infect. Dis. 17, 479-484 (2013)

4. Alizadeh, S., Baleanu, D., Rezapour, S.: Analyzing transient response of the parallel RCL circuit by using the Caputo-Fabrizio fractional derivative. Adv. Differ. Equ. 2020, 55 (2020). https://doi.org/10.1186/s13662-020-2527-0

5. Aydogan, S.M., Baleanu, D., Mousalou, A., Rezapour, S.: On high order fractional integro-differential equations including the Caputo-Fabrizio derivative. Bound. Value Probl. 2018, 90 (2018)

6. Rezapour, S., Samei, M.E.: On the existence of solutions for a multi-singular pointwise defined fractional q-integro-differential equation. Bound. Value Probl. 2020, 38 (2020). https://doi.org/10.1186/s13661-020-01342-3

7. Samei, M.E., Rezapour, S.: On a system of fractional q-differential inclusions via sum of two multi-term functions on a time scale. Bound. Value Probl. 2020, 135 (2020). https://doi.org/10.1186/s13661-020-01433-1

8. Baleanu, D., Mousalou, A., Rezapour, S.: On the existence of solutions for some infinite coefficient-symmetric Caputo-Fabrizio fractional integro-differential equations. Bound. Value Probl. 2017(1), 145 (2017). https://doi.org/10.1186/s13661-017-0867-9

9. Baleanu, D., Etemad, S., Rezapour, S.: On a fractional hybrid integro-differential equation with mixed hybrid integral boundary value conditions by using three operators. Alex. Eng. J. (2020). https://doi.org/10.1016/j.aej.2020.04.053

10. Baleanu, D., Nazemi, Z., Rezapour, S.: Attractivity for a k-dimensional system of fractional functional differential equations and global attractivity for a k-dimensional system of nonlinear fractional differential equations. J. Inequal. Appl. 2014, 31 (2014). https://doi.org/10.1186/1029-242X-2014-31

11. Ghorbanian, R., Hedayati, V., Postolache, M., Rezapour, S.: Attractivity for a k-dimensional system of fractional functional differential equations and global attractivity for a k-dimensional system of nonlinear fractional differential equations. J. Inequal. Appl. 2014, 319 (2014). https://doi.org/10.1186/1029-242X-2014-319

12. Agarwal, R.P., Baleanu, D., Hedayati, V., Rezapour, S.: Two fractional derivative inclusion problems via integral boundary conditions. Appl. Math. Comput. 257, 205-212 (2015). https://doi.org/10.1016/j.amc.2014.10.082

13. Alsaedi, A., Baleanu, D., Etemad, S., Rezapour, S.: On coupled systems of time-fractional differential problems by using a new fractional derivative. J. Funct. Spaces 2016, Article ID 4626940 (2016). https://doi.org/10.1155/2016/4626940

14. Baleanu, D., Hedayati, V., Rezapour, S.: On two fractional differential inclusions. SpringerPlus 5, 882 (2016). https://doi.org/10.1186/s40064-016-2564-z

15. Aydogan, S.M., Nazemi, Z., Rezapour, S.: Positive solutions for a sum-type singular fractional integro-differential equation with m-point boundary conditions. Sci. Bull. "Politeh." Univ. Buchar., Ser. A, Appl. Math. Phys. 79(1), 89-98 (2017)

16. Etemad, S., Rezapour, S., Samei, M.E.: On a fractional Caputo-Hadamard inclusion problem with sum boundary value conditions by using approximate endpoint property. Math. Methods Appl. Sci. (2020). https://doi.org/10.1002/mma.6644

17. Aydogan, S.M., Baleanu, D., Aguilar, J.F.G., Rezapour, S., Samei, M.E.: Approximate endpoint solutions for a class of fractional q-differential inclusions. Fractals 26(8), 1-18 (2020). https://doi.org/10.1142/S0218348X20400290

18. Shiri, B., Wu, G.C., Baleanu, D.: Collocation methods for terminal value problems of tempered fractional differential equations. Appl. Numer. Math. 156, 385-395 (2020). https://doi.org/10.1016/j.apnum.2020.05.007 
19. Baleanu, D., Shiri, B.: Collocation methods for fractional differential equations involving non-singular kernel. Chaos Solitons Fractals 116, 136-145 (2018)

20. Shiri, B., Baleanu, D.: System of fractional differential algebraic equations with applications. Chaos Solitons Fractals 120, 203-212 (2019). https://doi.org/10.1016/j.chaos.2019.01.028

21. Ma, C.Y., Shiri, B., Wu, G.C., Baleanu, D.: New signal smoothing equations with short memory and variable order. Optik 218, 164507 (2020). https://doi.org/10.1016/j.ijleo.2020.164507

22. Kumar, D., Singh, J., Baleanu, D.: On the analysis of vibration equation involving a fractional derivative with Mittag-Leffler law. Math. Methods Appl. Sci. 43(1), 443-457 (2019)

23. Kumar, D., Singh, J., Tanwar, K., Baleanu, D.: A new fractional exothermic reactions model having constant heat source in porous media with power, exponential and Mittag-Leffler laws. Int. J. Heat Mass Transf. 138, 1222-1227 (2019). https://doi.org/10.1016/j.ijheatmasstransfer.2019.04.094

24. Singh, J.: Analysis of fractional blood alcohol model with composite fractional derivative. Chaos Solitons Fractals 140 , 110127 (2020). https://doi.org/10.1016/j.chaos.2020.110127

25. Baleanu, D., Aydogan, S.M., Mohammadi, H., Rezapour, S.: On modelling of epidemic childhood diseases with the Caputo-Fabrizio derivative by using the Laplace Adomian decomposition method. Alex. Eng. J. (2020). https://doi.org/10.1016/j.aej.2020.05.007

26. Baleanu, D., Rezapour, S., Mohammadi, H.: Some existence results on nonlinear fractional differential equations. Philos. Trans. R. Soc. Lond. A 2013, 371 (2013). https://doi.org/10.1098/rsta.2012.0144

27. Tuan, N.H., Mohammadi, H., Rezapour, S.: A mathematical model for Covid-19 transmission by using the Caputo fractional derivative. Chaos Solitons Fractals 140, 110107 (2020). https://doi.org/10.1016/j.chaos.2020.110107

28. Baleanu, D., Jajarmi, A., Mohammadi, H., Rezapour, S.: Analysis of the human liver model with Caputo-Fabrizio fractional derivative. Chaos Solitons Fractals 134, 109705 (2020)

29. Baleanu, D., Mohammadi, H., Rezapour, S.: Analysis of the model of HIV-1 infection of CD4+ T-cell with a new approach of fractional derivative. Adv. Differ. Equ. 2020, 71 (2020)

30. Baleanu, D., Rezapour, S., Saberpour, Z.: On fractional integro-differential inclusions via the extended fractional Caputo-Fabrizio derivation. Bound. Value Probl. 2019, 79 (2019)

31. Baleanu, D., Etemad, S., Rezapour, S.: A hybrid Caputo fractional modeling for thermostat with hybrid boundary value conditions. Bound. Value Probl. 2020, 64 (2020). https://doi.org/10.1186/s13661-020-01361-0

32. Dokuyucu, M.A., Celik, E., Bulut, H., Baskonus, H.M.: Cancer treatment model with the Caputo-Fabrizio fractional derivative. Eur. Phys. J. Plus 133, 92 (2018)

33. Khan, M.A., Hammouch, Z., Baleanu, D.: Modeling the dynamics of hepatitis E via the Caputo-Fabrizio derivative. Math. Model. Nat. Phenom. 14(3), 311 (2019)

34. Ucar, E., Ozdemir, N., Altun, E.: Fractional order model of immune cells influenced by cancer cells. Math. Model. Nat. Phenom. 14(3), 308 (2019)

35. Ullah, S., Khan, M.A., Farooq, M., Hammouch, Z., Baleanu, D.: A fractional model for the dynamics of tuberculosis infection using Caputo-Fabrizio derivative. Discrete Contin. Dyn. Syst. 13(3), 975-993 (2020)

36. Singh, J., Kumar, D., Baleanu, D.: A new analysis of fractional fish farm model associated with Mittag-Leffler type kernel. Int. J. Biomath. 13(2), 2050010 (2020)

37. Goswami, A., Singh, J., Kumar, D., Sunshila: An efficient analytical approach for fractional equal width equations describing hydro-magnetic waves in cold plasma. Phys. A, Stat. Mech. Appl. 524, 563-575 (2019)

38. Srivastava, H.M., Dubey, V.P., Kumar, R., Singh, J., Kumar, D., Baleanu, D.: An efficient computational approach for a fractional-order biological population model with carrying capacity. Chaos Solitons Fractals 138, 109880 (2020). https://doi.org/10.1016/j.chaos.2020.109880

39. Singh, J., Kilicmen, A., Kumar, D., Swroop, R., Ali, F.M.: Numerical study for fractional model of nonlinear predator-prey biological population dynamical system. Therm. Sci. 23(6), 2017-2025 (2019)

40. Khalid, M., Samikhan, F.: Stability analysis of deterministic mathematical model for Zika virus. Br. J. Math. Comput. Sci. 19(4), 1-10 (2016). https://doi.org/10.9734/BJMCS/2016/29834

41. Kibona, I.E., Yang, C.H.: SIR model of spread of Zika virus infections: Zikv linked to microcephaly simulations. Health 9(8), 1190-1210 (2017). https://doi.org/10.4236/health.2017.98086

42. Maysaroh, A., Waluya, S.B., Wuryanto: Analisis dan simulasi model matematika penyatik Zika dengan satu serotip virus Zika. UNNES J. Math. 8(1), 56-71 (2019)

43. Alkahtani, B.S.T., Atangana, A., Koca, l.: Novel analysis of the fractional Zika model using the Adams type predictor-corrector rule for non-singular and non-local fractional operators. J. Nonlinear Sci. Appl. 10, 3191-3200 (2017). https://doi.org/10.22436/jnsa.010.06.32

44. Samko, S.G., Kilbas, A.A., Marichev, O.I.: Fractional Integrals and Derivatives: Theory and Applications. Gordon \& Breach, Switzerland (1993)

45. Caputo, M., Fabrizio, M.: A new definition of fractional derivative without singular kernel. Prog. Fract. Differ. Appl. 1(2), 73-85 (2015)

46. Losada, J., Nieto, J.J.: Properties of the new fractional derivative without singular kernel. Prog. Fract. Differ. Appl. 1(2), 87-92 (2015)

47. Ullah, M.Z., Alzahrani, A.K., Baleanu, D.: An efficient numerical technique for a new fractional tuberculosis model with nonsingular derivative operator. J. Taibah Univ. Sci. 13(1), 1147-1157 (2019)

48. Gomez-Aguilar, J.F., Rosales-Garcia, J.J., Bernal-Alvarado, J.J., Cordova-Fraga, T., Guzman-Cabrera, R.: Fractional mechanical oscillators. Rev. Mex. Fis. 58, 348-352 (2012)

49. Van den Driessche, P., Watmough, J.: Reproduction numbers and subthreshold endemic equilibria for compartmental models of disease transmission. Math. Biosci. 180, 29-48 (2002)

50. Li, C., Zeng, F.: The finite difference methods for fractional ordinary differential equations. Numer. Funct. Anal. Optim. 34(2), 149-179 (2013) 